
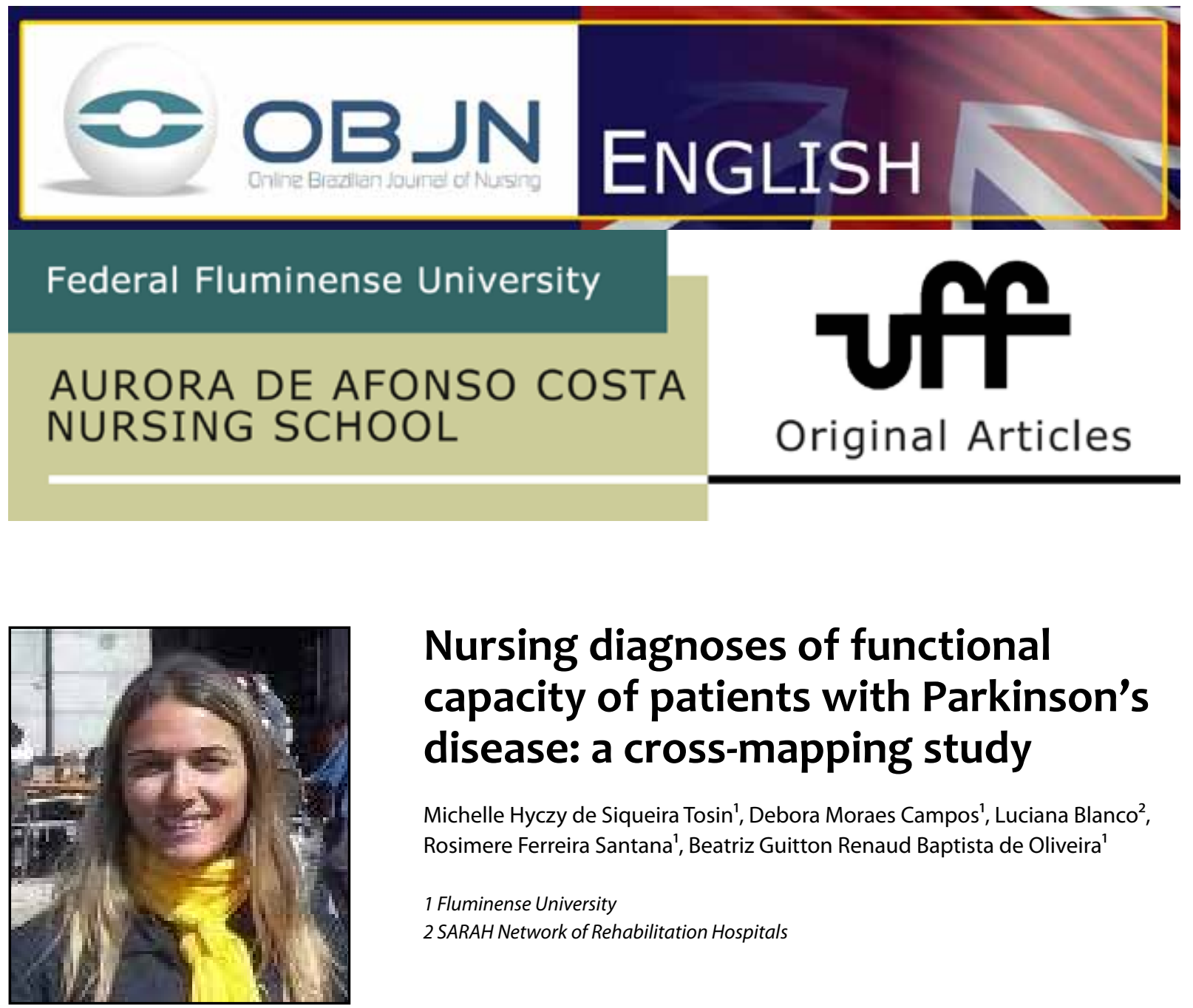

\title{
Nursing diagnoses of functional capacity of patients with Parkinson's disease: a cross-mapping study
}

\author{
Michelle Hyczy de Siqueira Tosin', Debora Moraes Campos', Luciana Blanco², \\ Rosimere Ferreira Santana', Beatriz Guitton Renaud Baptista de Oliveira' \\ 1 Fluminense University \\ 2 SARAH Network of Rehabilitation Hospitals
}

\section{ABSTRACT}

Aim: To carry out a cross mapping of the nursing diagnoses not standardized with the NANDA International classification system, present in the domains "Activity/rest" and "Safety/protection" and related to the functional capacity of patients with Parkinson's disease in a rehabilitation program. Method: a descriptive study with documentary research for the development of a cross-mapping. Simple random sample of 67 medical records and 335 nursing registers, from March 2009 to April 2013. Results: 15 NANDA International standardized diagnostics emerged from the cross mapping of the 93 terms of the nursing language. The most mapped terms were "Impaired physical mobility" (22\%) and "Risk of falling" (11\%). For "Impaired physical mobility" seven defining characteristics were mapped. Two terms of nursing language could not be mapped through the taxonomy. Conclusion: The cross mapping identified the standardized diagnoses related to the NANDA International. This enables the universalization and the consolidation of the role of nurses in Parkinson's disease rehabilitation.

Descriptors: Nursing Diagnosis; Rehabilitation; Parkinson Disease; Mobility limitation. 


\section{INTRODUCTION}

Parkinson's disease (PD) is a neurodegenerative disorder, with increasing incidence in the world population, which affects 93 individuals per hundred thousand inhabitants in the age group 50-59 years. This number increases to $5 \%$ in the elderly population from 70 years of age ${ }^{(1)}$. It is a disease with variable progression and poorly defined causal hypotheses, and is related to imbalances in neurotransmitter mechanisms in the central nervous system $^{(2)}$. Its manifestations are characterized by motor symptoms, such as rigidity, resting tremor, slowness of movement (bradykinesia) and postural instability; and non-motor symptoms, such as neuropsychiatric disorders, bowel, sexual and urinary systems ${ }^{(1-3)}$.

Since the original description of the disease, there have been radical changes in its concept, starting with a better understanding of the pathological manifestations and their drug therapy as well as of the diagnostic criteria ${ }^{(1)}$. However, advances of research indicate the need for constant updating of practice to ensure the care provided to the population. In this sense, understanding the pathophysiological and pharmacotherapeutic aspects of PD is essential for the development of the nursing care plan for the individual affected by the disease. These factors influence their functional capacity and negatively affect their quality of life. Recent studies reveal that living with PD strongly affects everyday life ${ }^{(4)}$. The experience of loss, decreased independence, and self-esteem, are the most referred-to themes ${ }^{(4)}$.

Therefore, the implementation of the nursing process, using the chosen taxonomy, should facilitate the diagnostic reasoning for prescribing care and the transmission of information between the nursing staff and researchers. In this point of view, the use of the
North American Nursing Diagnosis Association international classification system, known as NANDA International (NANDA-I), to define the nursing diagnoses described by nurses who work with the rehabilitation of patients with PD, allows the identification of the care needs for this population. This can be achieved due to the magnitude afforded by the use of this technological tool. Its taxonomic structure, represented in 13 domains, 47 classes and 201 nursing diagnoses, are arranged in an organized and synthesized way, based on the theoretical concepts of Gordon's functional health standards $s^{(5-7)}$.

Thus, the use of NANDA-I taxonomy in the nursing process provides conditions for improving nursing care in rehabilitation for patients with Parkinson's disease, as these allow the communication and comparison of nursing data across different contexts, countries and languages. Moreover, we observe that there is a scarcity of studies related to the issue in this very specific population ${ }^{(8-9)}$.

In this way, the results from this study may be used to support clinical decisions and diagnostic construction regarding the rehabilitation context related to the functional capacity of patients with PD.

Given the above, this study aims to conduct the cross mapping of the not-standardized nursing diagnoses which are present in the domains "Activity/rest" and "Safety/protection" and are related to the functional capacity of patients with Parkinson's disease in a rehabilitation program and the NANDA International classification system standard diagnoses.

\section{METHOD}

An applied-nature study, with a quantitative approach and descriptive purpose, with 
desk research as technical procedure, using charts as a primary source of data collection. As theoretical and methodological framework, we used the concepts and principles of cross mapping. This was chosen to linguistically and semantically compare the not-standardized terminology with the chosen classification system ${ }^{(8-10)}$.

The study - was conducted at the International Center for Neurorehabilitation and Neuroscience in the city of Rio de Janeiro. This center treats children and adults with neurological sequelae resulting from congenital or acquired lesions of the central nervous system. The rehabilitation team is multidisciplinary and the proposed treatment includes interventions, monitoring of the patient's rehabilitation process and guidance to the family members, according to the particularities of each case.

Currently, the care provided to patients by nurses is recorded in electronic records in textual form. In these, the nurses describe the phenomena through terms contained in the language commonly used by the team: the non-standardized terms. For this research, the authors considered the excerpts of developments that contained those terms to identify "diagnostic contexts", since there is no specific field for description of nursing diagnoses in the electronic system records.

Between its opening in March 2009 and April 2013, 1,266 patients were admitted with the diagnosis of Parkinson's disease. Of these, 796 had registered nursing care in electronic records. For a sample definition, we included records with five or more nursing records and excluded those containing, in addition to the diagnosis of Parkinson's disease, other titles related to other related parkinsonian syndromes such as secondary Parkinsonism.

Thus, 148 records were selected. Based on this population, we calculated the sample by probability, as simple random type, using the following formula(11):

$$
n 0=\frac{1}{E 0^{2}} \quad n=\frac{N \cdot n 0}{\mathrm{~N}+\mathrm{n} 0}
$$

We considered in this case: 148 records = population size $(\mathrm{N}), 9 \%$ = tolerable sampling error (E0), 123 records = first approximation of the sample size (n0) and 67 records = sample size $(n)$

Thus, the sample consisted of 67 records, representing $45 \%$ of the total population, assuming a sampling error of $9 \%$. Of this total, the last five nursing documentation were selected for mapping, totaling 335 analyzed records.

The cross mapping was carried out in three steps:

1) Sample categorization, extraction and normalization of terms;

2) Segregation and comparison of non-standardized nursing diagnoses with NANDA-I;

3) Evaluation and refinement of the mapping.

In the first stage, we performed the complete electronic extraction of the information that made up the database: characterization of patients; medical diagnosis and stage of Parkinson's disease; excerpt regarding the exact diagnosis context, extracted from the nursing records; extraction of the terms of the nursing language that indicated diagnostic hypotheses (defining characteristics or risk factors). For example, in the passage of records which described that "patient complains of worsening rigidity, bradykinesia, and gait pattern", the words "bradykinesia" and "worsening gait" were highlighted by the fragmentation of the record excerpt. From the part describing that "patient has a history of falling from his own height", we extracted the term "history of falls". 
These data were stored in a spreadsheet through Microsoft Excel for Mac Version 15.11.2 software and subject to spelling correction, adequacy of verbal tenses, uniformity of gender and number, exclusion of repetitions, identification of synonyms of casual expressions that do not designate particular concepts $^{(8-9)}$.

In the next step, we proceeded to cross-mapping the terms identified in the previous step with the nursing diagnoses of NANDA-I taxonomy. To this end, the following rules of cross mapping were used: mapping the context of the terms and the meaning of words (and not just the words) ${ }^{(8-9)}$.

The terms of the nursing language were compared to the defining characteristics or risk factors of NANDA-I. The categorization of nursing terms was carried out through an adapted analysis of combination. When the term found matched exactly the one in the classification system, it was categorized as an exact combination; when this term had synonyms, similar concepts and related terms, it was considered a partial combination. Terms that did not show any resemblance to the classification system were not classified as a combination, but even so, were exposed for analysis.

For the presentation of analysis results, exact and partial combinations were considered with the same value. In the aforementioned examples, the terms "bradykinesia" and "worsening gait" (extracted by fragmenting the record excerpt, and considering the diagnostic context) were considered partial combinations of the defining characteristics of NANDA-I "slow motion" and "changes in gait" which led to the diagnosis of "impaired physical mobility". But the term "history of falls" was considered an exact combination of the NANDA-I risk factor, which led to the diagnosis of risk of falls.
The data collected in the second stage were organized according to diagnosis title, definition, terms of nursing language and standardized terms of NANDA-I which met clinical confirmatory evidence of the presence of diagnosis.

The third step is the evaluation and refinement of cross mapping. For this purpose, the data collected were analyzed by experts: two specialist nurses in Nursing Classification and three in Parkinson's disease. For the selection of experts, we considered as the minimum requirements practical experience of five years or a doctorate and experience in research on nursing terminologies. The experts stated their agreement or disagreement concerning the relationship established by the authors between diagnoses titles, the terms of Nursing language and NANDA-I defining characteristics or risk factors. This step was performed in two cycles: individual and group. No new cycles were required as there was full consensus of the evaluators in the second one. This fact exempted the need for analysis of statistical agreement among the experts, previously scheduled for this research.

With regard to nursing diagnoses, 93 standard terms were identified, extracted from the excerpts of the nursing records considered by the authors as diagnostic contexts.

The development of this study met the national and international standards of ethics in research involving human beings ${ }^{(12)}$, since the sources of the results were the log records of patients.

\section{RESULTS}

The sample profile consisted of $63 \%$ of male patients with a mean age of 69.3 years $( \pm 10)$. The progression of Parkinson's disease 
ranged from 1 to 24 years. It was also found that $75 \%$ of them had 1-8 years of evolution of PD and only $4.5 \%$ were in more advanced stages (between 17 and 24 years).

With regard to nursing diagnoses, after the cross-mapping with the taxonomy and the refinement done by the experts, the terms of nursing language resulted in 15 standardized diagnoses according to NANDA-I (Table 1).

Table 1 - NANDA-I diagnoses related to the functional capacity of patients with Parkinson's disease in a rehabilitation program $(n=67)$. Rio de Janeiro, 2014.

\begin{tabular}{|c|c|c|c|}
\hline $\begin{array}{l}\text { NANDA-I } \\
\text { Area }\end{array}$ & $\begin{array}{c}\text { Code: NANDA-I Nursing } \\
\text { diagnoses }\end{array}$ & $\mathrm{n}$ & $\%$ \\
\hline \multirow[t]{12}{*}{$\begin{array}{l}\text { Activity/ } \\
\text { rest }\end{array}$} & $\begin{array}{c}\text { 00085: Impaired physical } \\
\text { mobility }\end{array}$ & 20 & 30 \\
\hline & $\begin{array}{l}\text { 00200: Decreased heart tissue } \\
\text { perfusion risk }\end{array}$ & 16 & 24 \\
\hline & $\begin{array}{l}\text { 00201: Decreased brain tissue } \\
\text { perfusion risk }\end{array}$ & 16 & 24 \\
\hline & 00198: Impaired sleep pattern & 8 & 12 \\
\hline & $\begin{array}{l}\text { 00108: Self-care impairment } \\
\text { for bath }\end{array}$ & 7 & 10 \\
\hline & 00088: Walking impairment & 3 & 4 \\
\hline & $\begin{array}{l}\text { 00165: Improved willingness } \\
\text { to sleep }\end{array}$ & 3 & 4 \\
\hline & $\begin{array}{l}\text { 00109: Self-care impairment } \\
\text { for dressing }\end{array}$ & 2 & 3 \\
\hline & $\begin{array}{l}\text { 00090: Impaired transfer } \\
\text { capacity }\end{array}$ & 2 & 3 \\
\hline & 00096: Sleep deprivation & 1 & 1 \\
\hline & $\begin{array}{l}\text { 00091: Impaired mobility in } \\
\text { bed }\end{array}$ & 1 & 1 \\
\hline & $\begin{array}{l}\text { 00204: Ineffective peripheral } \\
\text { tissue perfusion }\end{array}$ & 1 & 1 \\
\hline \multirow{2}{*}{$\begin{array}{l}\text { Safety/pro- } \\
\text { tection }\end{array}$} & 00155: Risk of falls & 10 & 15 \\
\hline & 00046: Impaired skin integrity & 3 & 4 \\
\hline
\end{tabular}

Source: authors' study

We can observe that in the "Activity/rest" domain, 80 nursing diagnoses with a non-standard classification system were mapped. After the cross-mapping with NANDA-I taxonomy and refinement made by the experts, these resulted in 13 nursing diagnoses. This represents $86 \%$ of diagnoses present in the two domains of NANDA-I analyzed in this study. We also emphasize that the diagnostic set was present in $100 \%(n=67)$ of the records analyzed, represented by at least one diagnosis.

The most mapped NANDA-I nursing diagnoses, which are also directly related to functional capacity, were "Impaired physical mobility" (30\%) and "Bathing self-care deficit" (10\%). It is noteworthy, however, that although the diagnoses compromise the functional capacity of the individual, they are not explicitly related: "Risk for decreased cardiac tissue perfusion" and "Risk for ineffective cerebral tissue perfusion", were equally represented by $24 \%$ of the total. Likewise, the quality of sleep is also highlighted as "Disturbed sleep pattern" (8\%) and "Sleep deprivation" (1\%).

In the domain "Safety/protection", the cross mapping allowed the identification of 13 not-standardized nursing diagnoses; after the intersection with NANDA-I taxonomy, it resulted in only two (14\%). In this area, the diagnosis "Risk for falls" (10\%) and "Impaired skin integrity" (3\%) were mapped.

For a better understanding of the diagnosis "Impaired physical mobility", which was further mapped in this study, the 32 mapped defining characteristics are presented below in figure 1 . We observe that $53 \%$ were associated with gait problems. We also note that the defining characteristics "gait changes" (53\%), "slow motion" (19\%) and "limited ability to perform gross motor skills" (13\%) were repeated in the nursing records, because the same patient presented associated features. This explains its greater number relative to the total number of the "Impaired Physical Mobility" diagnosis itself. 
Table 2 - Defining characteristics of "Impaired Physical Mobility" diagnosis, according to NANDA-I system, identified in log records of patients with Parkinson's disease $(n=32)$. Rio de Janeiro, 2014.

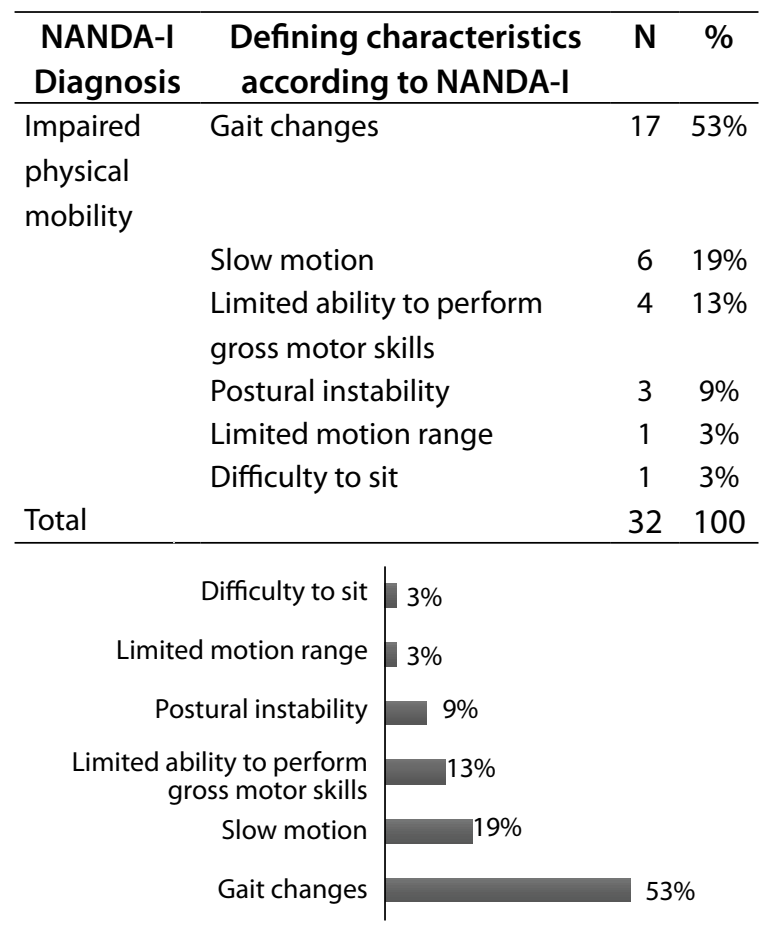

Two diagnosis contexts, present in 25 records, which could not be subject to cross mapping with NANDA-I taxonomy were also obtained (Table 2).

Table 3 - Diagnostic contexts identified in log records of patients with PD which could not be mapped using NANDA-I. Rio de Janeiro, 2014.

\begin{tabular}{lcc}
\hline \multicolumn{1}{c}{ Diagnostic contexts identified } & n & \% \\
\hline Independent for daily activities & 16 & 24 \\
Safe and efficient gait & 9 & 13 \\
Total & 25 & 37
\end{tabular}

Source: authors' study

\section{DISCUSSION}

Despite the methodological limitations of cross mapping, which is expected in the retrospective data collection by different professionals, the reliability and internal validity of the results of this research are given by the characteristics of the study site. In this, professionals are trained uniformly, the records are standardized and detailed, and patients are defined by specialty. Furthermore, we understand that the error adoption of $9 \%$ for the sample calculation can be regarded as a downside, since the usually adopted maximum error is $5 \%$. However, this is weighted by the multiplicity of nursing records that were obtained in each chart, which gave researchers a long data collection.

With regard to the characteristics of the sample, the findings converge with studies showing that PD tends to occur more frequently in men, especially in the age group over 60 years of age ${ }^{(1,2)}$. In this sense, we highlight aging itself, which can be considered an individual process that leads to progressive and irreversible deficits in functional systems. These can be represented by visual, auditory, motor and intellectual limitations as well as the emergence of chronic degenerative diseases, causing dependence in daily activities ${ }^{(13)}$. The intensification of these limitations can be present in patients with $\mathrm{PD}$, which has motor and non-motor abnormalities inherent to the pathophysiology of the disease itself $f^{(1,2)}$.

With regard to the time of disease progression in patients in the sample, there is growing evidence that the progression of PD is not linear and is directly related to individual aspects ${ }^{(14)}$. However, we observed that the degradation rate is much faster in the early stage of the disease, thus leading to functional impairment of the patients, who must be evaluated taking into account their individual characteristics ${ }^{(14)}$.

Therefore, the data regarding the sample categorization reveals that nurses work with patients in their daily life in old age and 
during a clear process of disease progression. So, when we think about the continuity, inherent to rehabilitation, performing the nursing process and the recording of gathered data is crucial for assessing disease progression. Similarly, it will ensure the planning of care in an assertive and grounded way.

With regard to nursing diagnoses, we observed during the analysis of records for data collection that the diagnostic stage of the nursing process was developed by the nurses. We also realized that the professionals were integrated into the rehabilitation interdisciplinary team, since the care were made both individually and as a team.

Among the most mapped nursing diagnoses in the domain "Activity/rest", "Impaired physical mobility" stands out. According to NANDA-I, impaired physical mobility is defined as the "limitation on independent and voluntary physical movement of the body or of one or more of its limbs"(6:283).

The loss of functional capacity that can be experienced by the patient is noteworthy in this diagnosis. The potential the patients have to decide and act independently in their lives is understood as functional capacity ${ }^{(15)}$.

Thus, it is observed that the patient with impaired physical mobility may have limitations directly related to the diagnostic "Bathing self-care deficit", "Impaired walking”, "Dressing self-care deficit", "Impaired transfer ability" and "Impaired bed mobility". The "Risk for falls", present in the domain "Safety/protection", can also be related to the diagnosis "Impaired physical mobility", both in the change in gait pattern, so present in PD patients, and in the changes in the postural stability ${ }^{(16)}$. Moreover, the age characteristic of patients in this study can be left aside because it is known that in the elderly the risk for falls is increased compared to young adults ${ }^{(17)}$.
The information generated by the assessment of functional capacity allows us to know the profile of the patients. In this sense, the use of the NANDA-I classification system makes it possible to determine the plan of care based on scientific evidence. Therefore we recognize the need to evaluate the defining characteristics of each diagnosis as a basis for the clinical reasoning of nurses because it culminates in ensuring diagnostic accuracy, enabling the most appropriate plan of care to be provided to patients with PD in the rehabilitation context ${ }^{(9)}$.

The diagnosis "Risk for decreased cardiac tissue perfusion" and "Risk of ineffective cerebral tissue perfusion" may indirectly compromise the functional capacity of the patients with PD. This occurs since the dysautonomia, defined as a dysfunction of the autonomic system that leads to postural hypotension, is a symptom that may be present in this population $^{(14)}$.

Studies reveals that this symptom is associated with dopaminergic therapy and with the failure of cardiovascular stimulation caused by dopaminergic denervation in myocardium $^{(14)}$. In this sense, the presence of hypotension may compromise patients' functional capacity and their safety in performing daily activities.

However, in the individual analysis of the records, there was a low percentage of hypotension as an associated risk factor. We observed that other diseases mentioned in the study as risk factors were present in these patients. Among these, hypertension and diabetes mellitus stand out. These morbidities can interfere indirectly with individual functionality, especially if its parameters are not controlled and are associated with complications such as sensorimotor polyneuropathy and vision changes. 
It is known that the prevalence of diabetic retinopathy after 15 years of disease progression varies from $97 \%$ in insulin-dependent diabetic patients and $80 \%$ in non-insulin dependent patients ${ }^{(18)}$. Also, polyneuropathy, as a disease with extensive pathological effect in the peripheral nervous system, can lead to sensory changes that may compromise the individual's functional ability in his proprioceptive mechanisms of gait ${ }^{(19)}$. Faced with this, we highlight the importance of defining the risk factors related to the nursing diagnosis as an indicator of diagnostic accuracy and hence the assertiveness of care measures being prescribed and implemented.

Other diagnoses that also call our attention in the domain "Activity/rest" are "Disturbed sleep pattern", "Readiness for enhanced sleep" and "Sleep deprivation". It is known that behavioral sleep disturbances are present in 40 to $50 \%$ of patients with $\mathrm{PD}^{(1)}$. They resonate directly and indirectly in all physiological and functional capacity of the individual, featuring a symptom of extreme importance to be identified and treated. The pathophysiological mechanisms of PD lead to various changes in sleep patterns: sleep fragmentation, REM sleep behavior disorder, excessive daytime sleepiness, altered sleep-wake cycle and sleep attacks induced by drugs ${ }^{(1)}$. The characterization of symptoms to define the nursing diagnosis is essential for its accuracy. This will enable the rehabilitation nurse to act on non-pharmacological aspects of treatment.

The nursing diagnosis "Impaired skin integrity", found in the domain "Safety/protection", had as the defining characteristic, according to NANDA-I, the disruption of skin surface. This diagnosis was recorded in the records of patients who, for coursing in advanced stages of PD, had pressure ulcers as a complication related to their functional deficit. Studies cite
PD as one of the causes of pressure ulcers in elderly patients with neurodegenerative diseases ${ }^{(20)}$. So, we can observe a reduction in physical fitness and functional capacity in patients in advanced stages of PD who present physical limitation and restriction to bed and wheelchair, requiring a careful investigation by the nurse of the risks of this complication in order to prevent and treat it if necessary.

For the diagnoses identified in nursing documentations that could not be mapped to the NANDA-I taxonomy, we highlight those related to health promotion. This happens because of diagnoses that cannot be defined as alterations and therefore are not subject to corrective measures, but to preventive care. The records showed that nurses seek for signs and symptoms that may interfere with the individual's functional capacity to perform daily activities such as walking and the ability to perform daily living activities. Even in their absence, they keep a register for longitudinal monitoring of these patients, who live with a progressive disease that can have motor fluctuations.

\section{CONCLUSION}

The cross mapping identified the NANDA-I diagnoses related to the functional capacity of patients with PD, through standardization of language.

The results from this study show a reduced percentage of diagnoses directly related to functional capacity. This may be related to the large number of patients who were in the early stages of the disease.

The sample size can be recognized as the main limitation of this research.

However, it appears that the use of the NANDA-I classification system may contribute 
to the progress of knowledge in this particular area of nursing work, since the standardization of language allows for the universalization and scientific consolidation of the praxis. In this context, we also highlight the diagnoses which could not be subject to mapping according to NANDA-I standards but are also an important point regarding progressive diseases with motor fluctuation character, as the PD.

\section{REFERENCES}

1. Olanow CW, Schapira AHV. Therapeutic Prospects for Parkinson Disease. Ann Neurol. 2013;74:337-47

2. Kim SR, So HY, Choi E, Kang JH, Kim HY, Chung SJ. Influencing effect of non-motor symptom clusters on quality of life in Parkinson's disease. J Neurol Sci. 2014;347:310-5.

3. Berg D, Postuma RB, Bloem B, Chan P, Dubois $B$, Gasser T, et al. "Time to redefine PD? Introductory statement of the MDS task force on the definition of Parkinson's Disease." Mov Dis. 2014;29(4):454-62.

4. Young-Mason J. The fine art of caring: people with Parkinson disease and their care partners. Clin Nurse Spec. 2015;29(2):121-2.

5. Souza JM, Veríssimo M. Child Development in the NANDA-I and international classification for nursing practices nursing classifications. Int J Nurs Knowl. 2013 Feb;24(1):44-8.

6. North American Nursing Diagnosis Association (NANDA International). Diagnósticos de enfermagem da NANDA: definições e classificação 2012-2014. Porto Alegre: Artmed; 2013.456 p.

7. Jones D, Duffy ME, Flanagan J, et al. Psychometric Evaluation of the Functional Health Pattern Assessment Screening Tool (FHPAST). Int J Nurs Knowl. 2012 Oct;23(3):140-5.

8. Tosin MH, Campos DM, Blanco L, Santana RF, Oliveira $B G$. Mapping nursing language terms of Parkinson's disease. Rev Esc Enferm USP. 2015 July;49(3):409-16.

9. Campos DM, Tosin MH, Blanco L, Santana RF, Oliveira BG. Nursing diagnoses for urinary disorders in patients with Parkinson's disease. Acta Paul Enferm. 2015;28(2):190-5.

10. Kim TY, Hardiker N, Coenen A. Inter-terminology mapping of nursing problems. J Biomed Inform. 2014;49:213-20.

11. Hulley SB, Cumming SR, Browner WS, Grady DG, Hearst NB, Newman TB. Delineando a pesquisa clínica: uma abordagem epidemiológica. 3a ed. Porto Alegre: Artmed; 2008.

12. Resolução n० 466 de 12 de dezembro de 2012. Publicada no DOU no 12 - quinta-feira, 13 de junho de 2013 - Seção 1 - Página 59. [site] Disponível em: http://conselho.saude.gov. br/resolucoes/2012/Reso466.pdf. Acesso em 22/03/2014.

13. Aires M, Paskulin LMG, Morais EP. Functional capacity of elder elderly: comparative study in three regions of Rio Grande do Sul. Rev latinoam enferm. 2010 Jan-Feb;18(1):11-7.

14. Fahn S, Jankovic J, Hallett M. Principles and practice of movement disorders. 2nd ed. New York: Elsevier; 2011.548p.

15. Castaneda L, Bergmann A, Bahia L. The International Classification of Functioning, Disability and Health: a systematic review of observational studies. Rev Bras Epidemiol. 2014 Apr-Jun; 17(2):437-51.

16. Hershey LA, Lichter DG. Freezing of gait in PD has a REM correlate: Twice cursed with a shared pathophysiology? J Neurol. 2013;81;1026-7.

17. Ebersbach G, Moreau C, Gandor F, Defebvre L, Devos D. Clinical syndromes: Parkinsonian gait. Mov Disord. 2013;28(11):1552-9.

18. Saldanha IJ, Dickersin K, Wang X, LiT. Outcomes in cochrane systematic reviews addressing four common eye conditions: an evaluation of completeness and comparability. PLoS One. 2014;9(10):1-10.

19. Guler EK, Eser I, Khorshid L, Yucel SC. Nursing diagnoses in elderly residents of a nursing home: a case in Turkey. Nurs Outlook. 2012;60:21-8.

20. Pereira AGS, Santos CT, Menegon DB, Mello B, Azambuja F, Lucena AF. Mapping the nursing care with the NIC for patients in risk for pressure ulcer. Rev Esc Enferm USP. 2014;48(3):454-61 
All authors participated in the phases of this publication in one or more of the following steps, in According to the recommendations of the International Committee of Medical Journal Editors (ICMJE, 2013): (a) substantial involvement in the planning or preparation of the manuscript or in the collection, analysis or interpretation of data; (b) preparation of the manuscript or conducting critical revision of intellectual content; (c) approval of the version submitted of this manuscript. All authors declare for the appropriate purposes that the responsibilities related to all aspects of the manuscript submitted to OBJN are yours. They ensure that issues related to the accuracy or integrity of any part of the article were properly investigated and resolved. Therefore, they exempt the OBJN of any participation whatsoever in any imbroglios concerning the content under consideration. All authors declare that they have no conflict of interest of financial or personal nature concerning this manuscript which may influence the writing and/ or interpretation of the findings. This statement has been digitally signed by all authors as recommended by the ICMJE, whose model is available in http://www. objnursing.uff.br/normas/DUDE_eng_13-06-2013.pdf
Received: $12 / 16 / 2014$

Revised: 09/14/2015

Approved: 09/16/2015

Tosin MHS, Campos DM, Blanco L, Santana RF, Oliveira BGRB. Nursing diagnoses of functional capacity of patients with 